\title{
On the Design of a Thermal Display for Upper Extremity Prosthetics
}

\author{
Keehoon Kim* $\quad$ J. Edward Colgate ${ }^{\dagger} \quad$ Michael A. Peshkin
}

Department of Mechanical Engineering, Northwestern University, USA

\begin{abstract}
We are developing a multi-function haptic device that displays pressure, vibration, shear force, and temperature to the skin of upper extremity amputees, especially those who have undergone targeted nerve reinnervation (TRI) surgery. This paper presents the design of the thermal display portion of this device. Through psychophysical temperature discrimination tests, the unique thermal response characteristics of intact hands and TRI-chests are explored.
\end{abstract}

Index Terms: H.5.2 [INFORMATION INTERFACES AND PRESENTATION]: User Interfaces—Haptic I/O

\section{INTRODUCTION}

The importance of restoring thermal sensation to upper extremity amputees is often considered minimal due to its limited functional role. In other words, thermal sensation is not critical to most activities of daily living (ADLs). But thermal sensation is important socially and emotionally. Amputees often express the desire to feel the warmth of a loved one's hand, or the sensation of holding a warm cup of coffee. Thus, as a component of a larger effort aimed at restoring haptic sensation to those who have lost an arm or hand, we are exploring thermal display.

This work is most relevant in the context of patients who have undergone "targeted reinnervation" (TRI) surgery [1-3]. TRI creates sensory spots on a patient's skin (for instance the chest or upper arm) that are perceived as spots on the skin of the phantom hand. Thus, by placing sensors on the prosthetic hand and using these to command tactors on the reinnervated skin, it should be possible to create a realistic sense of touch.

Since the tactor is attached to the body during daily life, it should be light weight and aesthetic and it should consume little power. Additionally, since the spatial resolution of the sensation created by the tactor depends on its size, it should be designed to be as compact as possible so that multiple tactors can be placed in the nerve reinnervated region.

Recently, a number of thermal tactile displays, the vast majority based on thermoelectric (peltier) devices, have been reported in the literature [4-6]. The devices described in the literature have limited application to our problem, however, due to the constraints of size and power. Most designs assume that i) the peltier device has enough power to control the temperature of the contact surface and ii) the thermal systems have an infinite heat sink. Since both the peltier device and heat sink are size-limited in our situation, we need to consider factors such as the patient's perception of small temperature changes as well as heat flux in and out of the heat sink.

In section 2, we begin with a brief introduction of the overall characteristics of the tactor including mechanical haptic display portion. In order to determine the elements of the thermal display, we investigated the quantitative specifications based on the literature and an experiment with a TRI patient. This design procedure

\footnotetext{
*e-mail: keehoon-kim@northwestern.edu

†e-mail: colgate@northwestern.edu

†e-mail: peshkin@northwestern.edu
}

Symposium on Haptic Interfaces for Virtual Environments and Teleoperator Systems 2008

13-14 March, Reno, Nevada, USA

978-1-4244-2005-6/08/\$25.00 @2008 IEEE

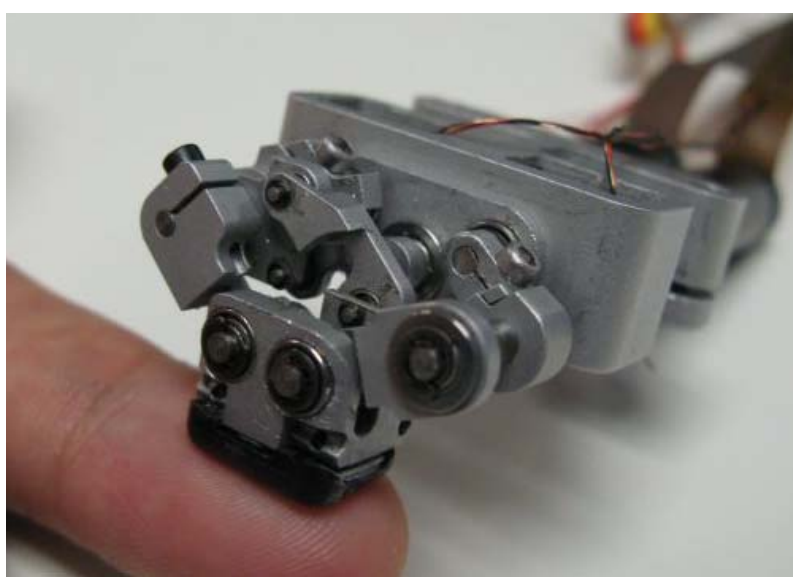

Figure 1: TACTOR20: a multi function tactor which can display contact, pressure, vibration, shear force, and temperature.

will be shown in Section 3. We have also performed a psychophysical study of temperature perception with non-TRI subjects. The results indicate a consistent pattern as will be explained in section 4. This section also discusses preliminary experimental results with TRI patients. This is followed by conclusions in section 5 .

\section{BRIEF INTRODUCTION OF TACTOR}

Because haptics comprises such an extraordinarily rich set of sensory pathways, it was important for our team to prioritize those which we wished to restore. Discussion among team members ${ }^{1}$ led to the prioritization of restoring sensation: i) contact, ii) pressure, iii) vibration, iv) temperature, v) shear force, and vi) fine shape discrimination.

For the first prototype, we have built a multi-function tactor that can display contact, pressure, vibration, shear force, and temperature simultaneously as shown in Fig.1.

In this section, we will introduce the mechanical portion of the tactor. The tactor design incorporates two degrees-of-freedom (DOF) to enable both pressure and shear. In addition, we felt it important to keep the tactor head parallel to the skin surface at all times. We considered a variety of mechanism designs for converting two actuator outputs to two translations of the tactor head, and ultimately settled on a 6 bar mechanism with two skewed parallelogram linkages. Because it involves no gears or sliding joints, this mechanism is low in both lost motion (e.g., backlash) and friction. Moreover, the skewed parallelograms help to maximize the workspace of the tactor by minimizing the singularity region. Since the tactor contains closed kinematic loops, we used carefully selected bearing configurations to avoid over-constraint problems caused by joint axes that are misaligned during assembly or manufacture. As a result, the tactor has $10 \mathrm{~mm}$ by $12 \mathrm{~mm}$ workspace and generates $9.0 \mathrm{~N}$ continuous force with isotropic manipulability. It is capable of generating vibration at least an order of magnitude

${ }^{1}$ We wish to acknowledge Prof. Ronald Johansson, members of Prof. Paolo Dario's Cyberhand group and Prof. Allison Okamura 


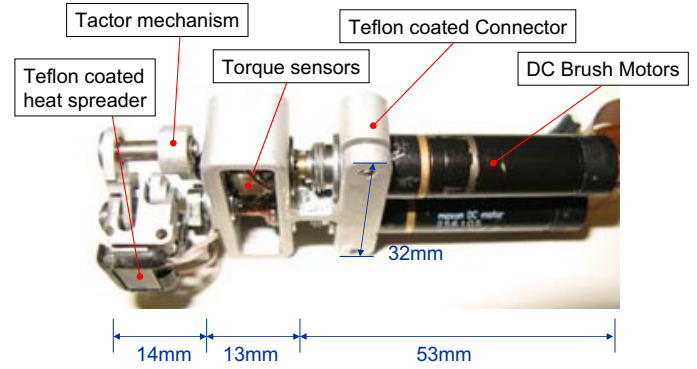

Figure 2: Dimensions of TACTOR20. Components that may come into contact with the skin are Teflon coated to minimize possible chafing or irritation

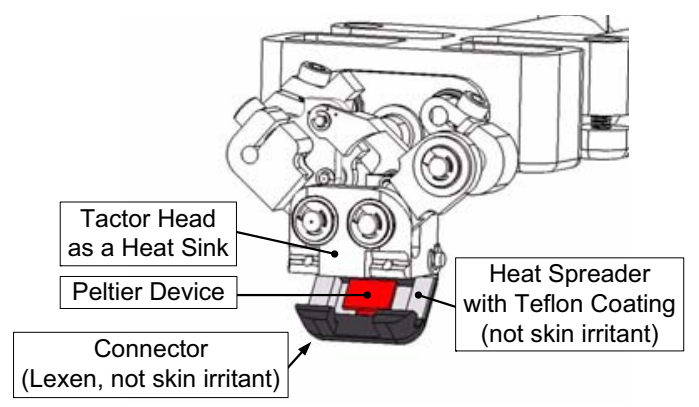

Figure 3: Components of the thermal display: tactor head as a heat sink, peltier device, and heat spreader

$(20 \mathrm{~dB})$ greater than the vibration detection thresholds for the finger as measured by Bolanowski et al. [7]. For closed loop force control, the tactor head would be an ideal place for a force sensor, but this region is reserved for the thermal display. Instead, we have developed miniature torque sensors. Using the conventional torque to force mapping, control of contact, pressure, and shear force have been successfully demonstrated [8].

The length and width of the tactor are $80 \mathrm{~mm}$ and $32 \mathrm{~mm}$ respectively, as shown in Fig.2. The height is changed by the pose of the tactor mechanism from $13 \mathrm{~mm}$ to $25 \mathrm{~mm}$. The mass of the tactor is 56 grams. Both mass and dimensions of the tactor significantly depend on the DC brushed motor actuators (64\% of total mass and $66 \%$ of total length). Exploring actuator alternatives is an important issue for future work.

\section{Design of the Thermal Display}

This section presents the key design decisions for the thermal display portion of the tactor. We address first how we selected the elements of the thermal display, and second the threshold test with a TRI patient used to size the heat spreader.

\subsection{Specification of Thermal Display}

Fig. 3 shows the configuration of a tactor head, peltier device, and a heat spreader. Because the peltier device and heat spreader are mounted to the most distal link of the tactor mechanism which we call the "tactor head", that link serves as a heat sink. For the peltier device, $5.2 \mathrm{~mm} \times 9.6 \mathrm{~mm}$ is allowed on the tactor head. The sizing of the heat spreader will be explained later.

In order to specify the perfomance of the thermal display, it is necessary to understand the heat flux requirements as well as the range of temperatures that must be displayed. Though the objective of the thermal display is to transfer a realistic thermal sensation to a patient, it is not desirable to display painful sensations. Therefore,
Table 1: Required specifications of the thermal display

$$
\begin{array}{c|c}
\text { Size of the thermal display } & 5.2 \mathrm{~mm} \times 9.6 \\
\text { Operation range } & 15 \sim 40^{\circ} \mathrm{C} \\
\text { Maximum heat flux } & 3.5 \times 10^{4} \mathrm{~W} / \mathrm{m}^{2}
\end{array}
$$

the operation range of temperature is chosen to be $15^{\circ} \mathrm{C}$ to $40^{\circ} \mathrm{C}$ [9] [10].

Ho and Jones have studied the heat flux between skin and various materials through simulations using a semi-infinite body model $[4,5]$. In order to differentiate a copper material ${ }^{2}$ from others under $1.4 \times 10^{4} \mathrm{~Pa}$ contact pressure between $24^{\circ} \mathrm{C}$ material and $32^{\circ} \mathrm{C}$ human skin, $3.05 \times 10^{4} \mathrm{~W} / \mathrm{m}^{2}$ heat flux power is required. Table 1 summarizes the required specification of the thermal display.

\subsection{Heat Flux Actuator and Temperature Sensor Selec- tion}

We selected a peltier device as the heat flux actuator due to its small size, ease of control, and ability to cool as well as heat. For our thermal display, we selected the TE-8-0.45-1.3 from TE technology.

In order to detect temperature, we considered heat flux sensors, thermistors, and thermocouples. Though the operation range is not a major consideration, response time, sensitivity, and size are important factors to satisfy the performance requirements. Table 2 shows several commercial sensors and their specifications. Thermistors have good resolution less than $0.01^{\circ} \mathrm{C}$ but slow time constants longer than $1.0 \mathrm{sec}$ so that it is difficult to implement closed loop control for a rapidly changing temperature profile. Heat flux sensors have the advantage of measuring the key control variable heat flux - directly. But these devices don't satisfy the size constraint and exhibit time constant longer than 0.6sec. The 5TCTT-J-40-36 thermocouple from OMEGA, has a very small size so that response time is less than $0.001 \mathrm{sec}$ if a 0.08 diameter spherical lumped model is assumed. However, in order to calculate the temperature from the thermocouple, it requires voltage amplification and temperature compensation. Nonetheless, we selected the thermocouple as a temperature sensor and the thermistor as a temperature compensator subject to the assumption of slow ambient temperature change.

\subsection{Heat Spreader Selection}

The surface of heat spreader contacts the human skin and transfers the heat flux from the peltier device to the skin. It has a smooth shape for comfort, and a larger area than the peltier device in order to enhance perception. It is known that temperature discrimination depends on the area of thermal stimulator [12]. Therefore, in order to determine an appropriate dimension for the heat spreader, a temperature discrimination threshold test was performed with a TRI patient.

For the experiment, 3 different sizes of heat spreader were used: $5.0 \mathrm{~mm}, 8.0 \mathrm{~mm}$, and $10.0 \mathrm{~mm}$ diameter. Fig. 4 shows the protocol for the experiment. Initially, since the amputee's skin temperature was $32^{\circ} \mathrm{C}$, the base temperature of the heat spreader was set to $32^{\circ} \mathrm{C}$. From the base temperature, the temperature was increased to a desired temperature in 1 second. The stimulation temperature was maintained for given stabilization time. In this experiment, two lengths of stabilization time were applied: 4.0 seconds and 8.0 seconds. The subject was given longer than 5 seconds between each stimulation. In order to search for the threshold temperature, a $0.5^{\circ} \mathrm{C}$ resolution was used.

\footnotetext{
${ }^{2}$ Among the materials simulated in the literature, copper results in the highest heat flux for a given temperature differential between it and human skin.
} 
Table 2: Commercial Temperature Sensor and Their specification

\begin{tabular}{c|c|c|c} 
product & resolution & time constant & dimension \\
\hline 44033 thermistor(OMEGA [11]) & $0.01^{\circ} \mathrm{C}$ & $>1.0 \mathrm{sec}$ & $2.4 \mathrm{~mm} \mathrm{diameter}$ \\
HFS-3 heat flux sensor(OMEGA) & $6.5 \mu \mathrm{V} / \mathrm{Btu} / \mathrm{Ft} \mathrm{t}^{2}-\mathrm{Hr}$ & $>0.6 \mathrm{sec}$ & $28.5 \times 35.1 \mathrm{~mm} \mathrm{~m}^{2}$ \\
5TC-TT-J-40-36 thermocouple(OMEGA) & $0.05 \mathrm{mV} /{ }^{\circ} \mathrm{C}$ in the operation range & $<0.001 \mathrm{sec}$ for $0.08 \mathrm{~mm}$ lumped model & $0.08 \mathrm{~mm}$ diameter
\end{tabular}

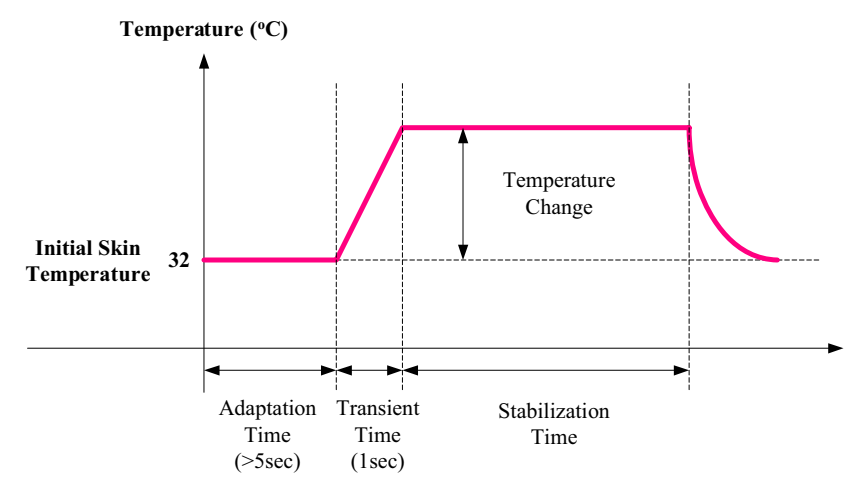

Figure 4: Protocol for the experiment of temperature discrimination using different size heat spreaders and a nerve re-innervated amputee.

Table 3: Temperature discrimination of a nerve re-innervated amputee for different stabilization times and heat spreader diameters.

\begin{tabular}{cc|c|c|c|c} 
& & \multicolumn{2}{|c|}{ Heating } & \multicolumn{2}{c}{ Cooling } \\
\hline Diameter & Stabilization time & $4 \mathrm{sec}$ & $8 \mathrm{sec}$ & $4 \mathrm{sec}$ & $8 \mathrm{sec}$ \\
\hline \multirow{2}{*}{$5 \mathrm{~mm}$} & $12^{\circ} \mathrm{C}$ & $12^{\circ} \mathrm{C}$ & $4.0^{\circ} \mathrm{C}$ & $4.0^{\circ} \mathrm{C}$ \\
& $8 \mathrm{~mm}$ & $8.5^{\circ} \mathrm{C}$ & $8.5^{\circ} \mathrm{C}$ & $5.0^{\circ} \mathrm{C}$ & $4.0^{\circ} \mathrm{C}$ \\
& $10 \mathrm{~mm}$ & $7.5^{\circ} \mathrm{C}$ & $7.0^{\circ} \mathrm{C}$ & $4.5^{\circ} \mathrm{C}$ & $4.5^{\circ} \mathrm{C}$
\end{tabular}

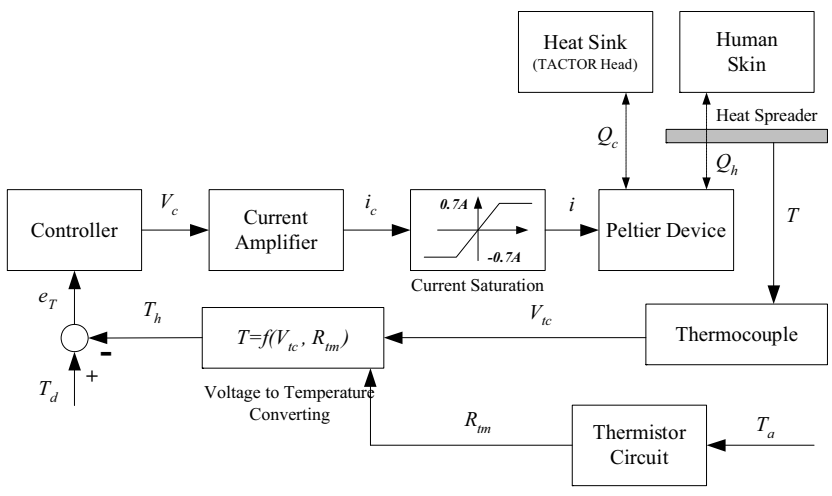

Figure 5: Temperature control block diagram.

Table 3 shows the results of the experiment. For heating, a larger diameter heat spreader leads to better discrimination. The stabilization time makes no difference. The subject felt the heating and cooling temperature immediately after the heat transient time. For cooling, discrimination threshold is not much affected by either the size of the heat spreader or stabilization time. Although this experiment suggests that a larger diameter heat spreader is desirable, a larger diameter also turns out to increase the power required for displaying pressure. Moreover, the improvement beyond $8.0 \mathrm{~mm}$ is modest; thus, an $8.0 \mathrm{~mm}$ diameter is thought to be close to optimal. For the heat spreader of the tactor, a $5.6 \times 9.6\left(\mathrm{~mm}^{2}\right)$ area (a size comparable to the 8.0 diameter heat spreader) and $0.5(\mathrm{~mm})$ thin aluminium 6061 has been chosen.

An FEM analysis was performed to determine whether the heat spreader would indeed spread the heat out, or whether there would be a "hot spot" underneath the peltier device (which is quite a bit smaller than the spreader). The spreader was assumed to be made of 6061 aluminium due to its high thermal conductivity. We should note that aluminium is a skin irritant, but in our design it is coated with a very thin layer of Teflon. The simulation confirmed that the spreader temperature would be quite uniform. A similar analysis showed that tactor head acting as the heat sink, would also have a highly uniform temperature.

In order to maximize the thermal conductivity, a heat spreader, a thermocouple, the peltier device, and the heat sink (tactor head) are connected using thermal compounds and epoxies (Arctic Silver 5 and Arctic Silver, Arctic Silver Inc.).

\subsection{Temperature Control}

Fig.5 shows a temperature control block diagram for the thermal display. $T_{d}$ and $T_{h}$ are the desired temperature and the measured skin temperature, respectively. A feedback controller is used to drive the peltier device with a current $i$ causing heat flow between the skin and heat sink as required to minimize the error $e_{T}$ between the desired and measured temperature. 


\section{Psychophysical Temperature Discrimination TEST}

In order to display thermal sensations to TRI patients, it is important to understand how they perceive temperature changes. In addition, since the sensory nerves that reinnervate their chest skin previously innervated their hands, we were interested in the following question: Is the perception of temperature change on the TRI chest closer to that of the non-TRI chest or to that of the non-TRI hand?

As a the first step toward answering these questions, we carried out a set of psychophysical tests with two TRI patients and several volunteer subjects having no known injury or perceptual deficit. This section describes the experiments and presents data for both TRI patients' chests and one patient's hand as well as the hands of five non-TRI subjects.

In the experiments, the following gaussian temperature profile was applied to the subjects' skin:

$$
T_{d}(t)=\left(T_{p}-T_{S}\right) \exp \left(-0.5(t-3 \sigma)^{2} / \sigma^{2}\right),
$$

where $T_{d}(t)$ is the desired temperature (See Fig.5), $T_{p}$ and $T_{s}$ are the peak temperature and the skin temperature, $\sigma$ represents the rate of temperature change, and $t$ is time and $0<t<6 \sigma$. The purpose of this temperature profile was to allow us to explore the effects of both absolute temperature change and rate of temperature change.

To ensure consistency, the following conditions were observed for each trial: 1) The temperature was carefully controlled to ensure that no actual tissue damage would occur, and subjects were free to remove the display or stop the experiment at any time. 2) The stimulation point was the spot \#1 in Fig.8. 3) Subjects were asked to classify the thermal stimulus on a 7 point Likert scale of sensation, i.e, 'feel nothing', 'warm-', 'warm', 'warm+', 'hot', 'hot' ,and 'hot+'. The last of these, 'hot+', indicates that the subject feels that any further heating might cause pain. 4) Subjects were trained to make their own 7 point Likert scale of thermal sensation for about 10 minutes. 5) At the beginning and end of each trial, auditory cues were presented to subjects, and any false positive answers were recorded. 6) Before starting the trials, we asked each subject put his or her hand on the tactor and waited until the tactor temperature converged to the subjects' skin temperature. 7) We recorded the skin temperature to know if the perception depends on the absolute temperature, $T_{p}$, or relative temperature, $T_{p}-T_{s} .8$ ) Each set of trials was completed in about 1.5 to 2 hours under IRB approval. We gave 2 to 3 minutes break time and let the subject take a rest whenever he or she wanted.

\subsection{Tests with Non-TRI Subjects}

A total of six healthy, naive subjects, three females and males aging from 26 to 34, were involved in this experiment. The subjects were free to select which hand they wanted use. During each trial, the subjects' skin temperature, $T_{s}$ in Eq.(1), varied between $26^{\circ} \mathrm{C}$ to $32^{\circ} \mathrm{C}$. After the training period, all subjects were confident that they had built consistent 7 point Likert scales. One subject's data were not consistent and a number of false positives were found. For the other five subjects, the data show consistency and there were no false positive answers.

Fig.6 shows the plots of the five consistent data sets. The plots show the responses of the subjects in 7 Likert scales in $\Delta T$ and $\sigma$ space, where $\Delta T=\left(T_{p}-T_{S}\right)$, i.e., the relative temperature.

Although it is difficult to draw any firm conclusions based on this limited number of subjects, certain features tend to emerge. Perhaps most interesting is the appearance of a critical value of $\sigma$ in several cases. The critical value is the value of $\sigma$ at which the subject is the most sensitive to temperature change. At lower values, the sensitivity decreases, presumably because the overall amount of heat being delivered to the finger is so small. At higher values the
Table 4: Curve Fitting for N03 Results (See Fig.6)

\begin{tabular}{l|c|c|c|c} 
Feeling & $\mathrm{a}$ & $\mathrm{b}$ & $\mathrm{c}$ & $\mathrm{RMS}$ \\
\hline$W-$ (warm-) & 0.3159 & 5.4806 & 11.2103 & 0.0498 \\
$W 0$ (warm) & 0.7830 & 15.2518 & 7.9105 & 0.1251 \\
$W+$ (warm+) & 0.8402 & 16.4773 & 9.5777 & 0.2945 \\
$H-$ (hot-) & 0.9059 & 32.5822 & 10.5154 & 0.1751 \\
$H 0$ (hot) & 0.2262 & 17.0957 & 19.7687 & 0.2720 \\
$H+$ (hot+) & -0.1512 & 0.5364 & 28.3406 & 0.1230
\end{tabular}

sensitivity also decreases for warm sensation rather than hot sensation, presumably because the thermal receptors adapt to the slowlychanging stimulus. The following model is a simple mathematical form capable of predicting the critical value.

$$
\Delta T=a \sigma+b / \sigma+c,
$$

where $a, b$, and $c$ are constants. Fig.6(c) shows the interpolated test results for subject $\mathrm{N} 03$. The $\mathrm{o}, \times,+, \square, \diamond$, and $\triangle$, marks are the real response data points of 'warm-', 'warm', 'warm+', 'hot', 'hot', 'hot+', respectively. Six contours show the best-fit curves using Eq.(2). Table 4 shows the numerical values of constants for the best-fit curves and the root mean square errors.

\subsection{Preliminary Test with TRI Subjects}

In the same manner, we have performed psychophysical temperature response tests with two TRI patients, SD01 and SD02. Both are shoulder level disarticulation patients. SD01 lost both arms secondary to electrical burns while working as a high-power lineman [1]. SD02 lost one arm due to a motorcycle accident [2]. Fig.7 shows the data plot of the subjects' 7 Likert scale responses in $\Delta T$ and $\sigma$. We should not, however, that these plots represent a total of only 30 to 40 trials with each subject, as compared to 80 to 100 trials for each non-TRI subject. The reduced number of trials is due to limited time with the TRI patients.

The results show that the thermal sensation regions of both TRI chests (Fig.7(a)-(b)) are narrower than those of the intact hand (Fig.7(c)). In addition, SD01's sensory region is narrower than SD02. There are two possibilities: 1) SD01's chest was burned from the accident altering thermal perception; 2) There are differences between SD01's and SD02's TRI surgeries. SD02 has a hybrid representation that encompasses both the intrinsic neural regeneration through the muscle (like SD01) and she also has reinnervation from neural conduits. For details, please see [1-3].

An interesting point is that the thermal sensitivity of SD02's intact hand is much higher than any of the non-TRI subjects' hand as shown in section 4.1. The sensitivity of SD02's chest is also much higher than that of SD01's chest consistently.

SD01's chest can be represented by Eq.(2) though the warm sensation is much narrower than the case of the intact hand. For SD02's intact hand and TRI chest, we couldn't find the pattern represented by the second term in Eq.(2).

One obvious result of these experiments is that the TRI chest has much narrower band of warm and hot sensation than the intact hand. Thus, we expect that it will be difficult to display a warm sensation to the chest since the sensation jumps from none to hot pain easily. We conclude that: 1) a precise thermal display is required; 2) a careful mapping method is required to display realistic thermal sensations to the TRI chest.

We also obtained subjective feedback from both TRI patients. Both patients described the hot sensation as spreading out \#1 region to \#2 region as shown in Fig.8. SD01 compared the thermal sensation to touching a warm or hot object wearing a thin glove. SD02 imagined that a hot object came from a long distance away 


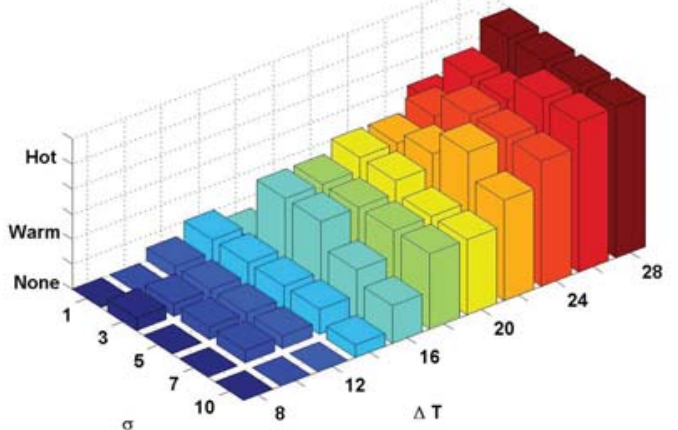

(a)

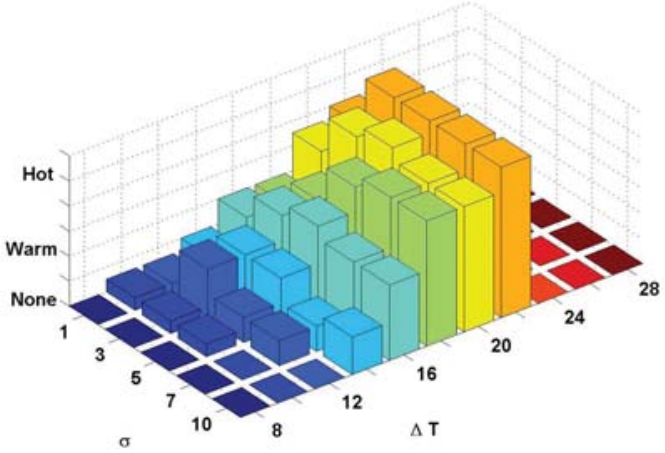

(b)

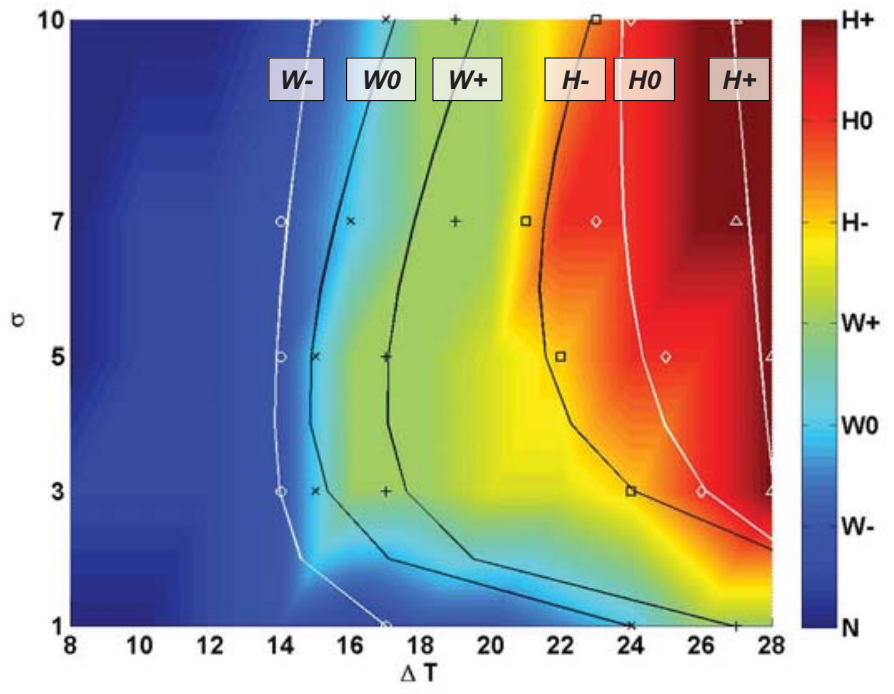

(c)

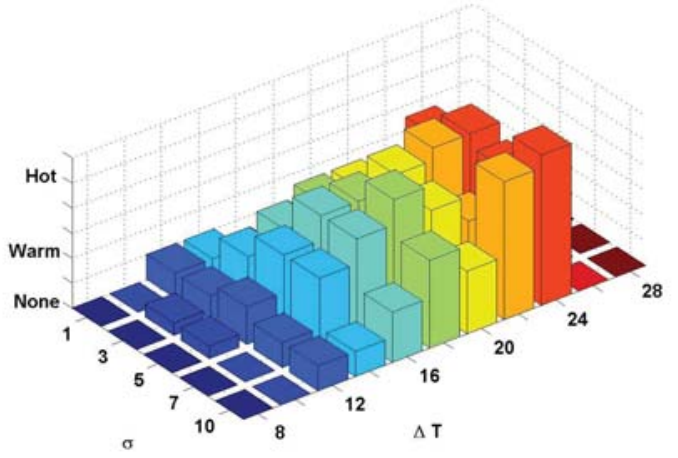

(d)

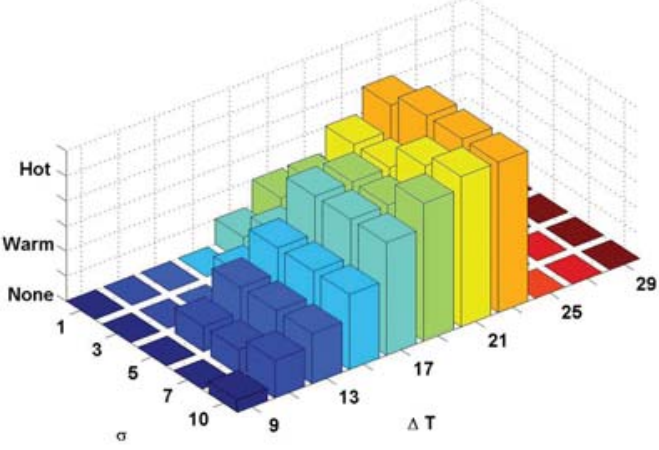

(e)

Figure 6: Experimental results of temperature discrimination tests (valid $\Delta T$ range): (a) $\mathrm{N} 01\left(8^{\circ} \mathrm{C}<\Delta T<28^{\circ} \mathrm{C}\right)\left(\right.$ b) $\mathrm{N} 02\left(8^{\circ} \mathrm{C}<\Delta T<22^{\circ} \mathrm{C}\right)$, $(\mathrm{c})$ N03 $\left(8^{\circ} \mathrm{C}<\Delta T<28^{\circ} \mathrm{C}\right)$, (d) N04 $\left(10^{\circ} \mathrm{C}<\Delta T<24^{\circ} \mathrm{C}\right)$, (e) N05 $\left(9^{\circ} \mathrm{C}<\Delta T<23^{\circ} \mathrm{C}\right)$ 


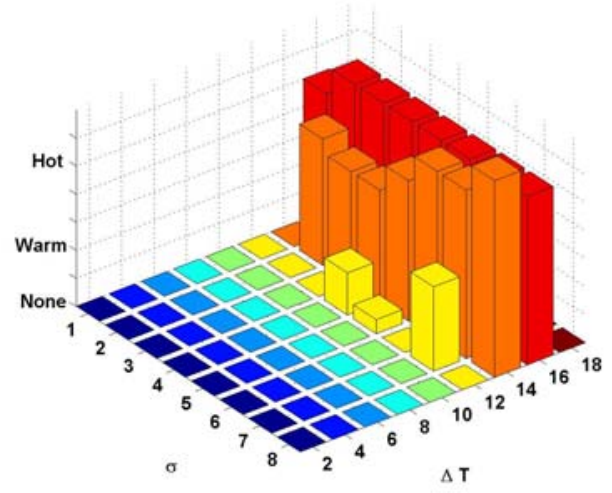

(a)

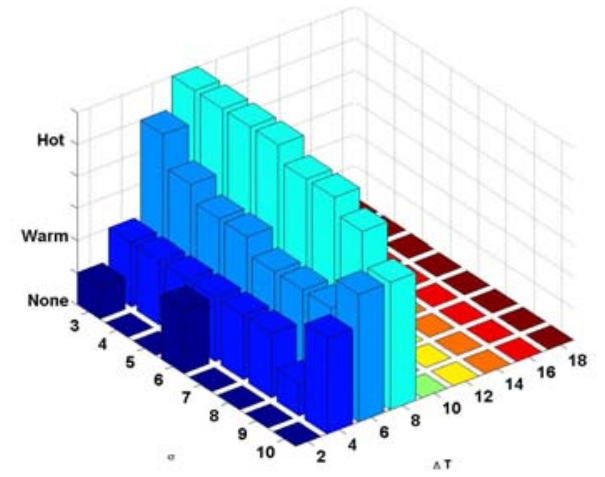

(b)

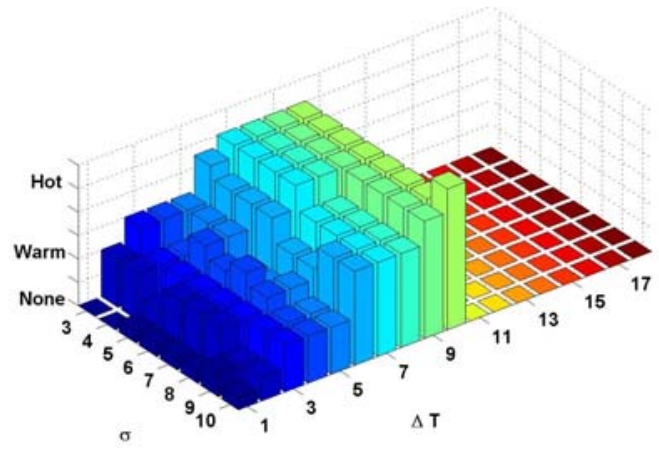

(c)

Figure 7: Experimental results of temperature discrimination tests with TRI subjects (valid $\Delta T$ range): (a) $\mathrm{SD} 01$ 's $\mathrm{TRI} \mathrm{Chest}\left(12^{\circ} \mathrm{C}<\Delta T<16^{\circ} \mathrm{C}\right.$ ), (b) SD02's TRI Chest $\left(2^{\circ} \mathrm{C}<\Delta T<8^{\circ} \mathrm{C}\right)$, (c) SD02's intact hand $\left(1^{\circ} \mathrm{C}<\Delta T<10^{\circ} \mathrm{C}\right)$ 


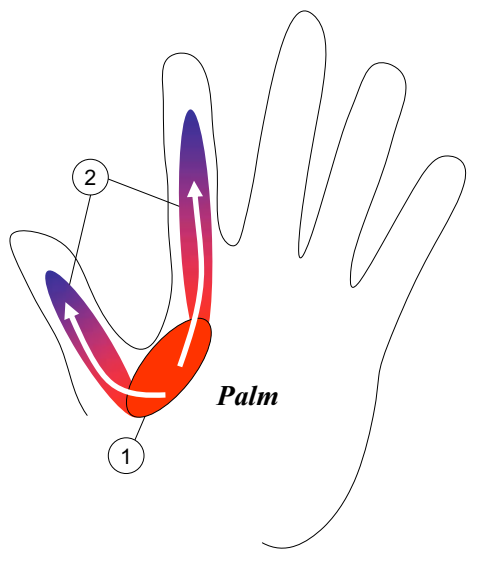

Figure 8: Shaded regions correspond to perceived location of stimulus on a phantom hand

to her hand but never actually touched the phantom hand. Thus, we tested two thermal display conditions using the tactor: 1) the heat spreader was allowed to heat up first and then touch her TRI chest, 2) the spreader was heated up after touching her chest. SD02 felt that the first condition was more realistic and there was no 'spreading out' feeling any more. It is apparent from these tests that will play an important role in realistic thermal display.

SD02 also commented that she felt scared of the thermal display on her chest and begin to feel the hot sensation quickly compared to her hand. It is quite possible that this resulted from different receptor populations in the chest and hand, and not from fear. Nonetheless, it is clearly important to establish trust in the safety of the tactor.

\section{Conclusion}

We are developing a tactor to display multi-modal haptic sensations. This paper presents the design of the thermal display portion. Although the thermal display has only 4 main components - a heat spreader, a peltier device, a thermocouple, and a heat sink - it requires careful design due to constraints such as limited size and power consumption.

We have performed psychophysical temperature discrimination tests on non-TRI subjects' hands as well as the chests and an intact hand of two TRI subjects. The results suggest a critical duration at which temperature sensitivity is greatest. They also indicate a much more rapid transition from no sensation to a strong heat sensation in the TRI chest than in the hand. These results provide useful insights into the design of thermal display algorithms, a topic of ongoing research.

\section{ACKNOWLEDGEMENTS}

This research was performed as part of the Johns Hopkins University Applied Physics Laboratory (JHUAPL) Revolutionizing Prosthetics 2009 funded by the Defense Advanced Research Projects Agency (DARPA) Contract \# N66001-06-C-8005. We also wish to thank Todd A. Kuiken, Laura A. Miller, Blair Lock, and Paul D. Marasco for their assistance and advice.

\section{REFERENCES}

[1] T. A. Kuiken, L. A. Miller, R. D. Lipschutz, K. A. Stubblefield, and G. A. Dumanian, "Prosthetic command signals following targeted hyper-reinnervation nerve transfer surgery," in Proc. of IEEE Enginerring in Medicine and Biology 27th Annual Conference, 2005.

[2] T. A. Kuiken, G. A. Dumanian, R. D. Lipschutz, L. A. Miller, and K. A. Stubblefield, "Targeted reinnervation for enhanced prosthetic arm function in a woman with a proximal amputation: a case study," vol. 369, pp. 371-380, Feb. 2007.

[3] T. A. Kuiken, L. A. Miller, R. D. Lipschutz, B. A. Lock, K. S. eld, P. D. Marasco, P. Zhou, and G. A. Dumanian, "The use of targeted muscle reinnervation for improved myoelectric prosthesis control in a bilateral shoulder disarticulation amputee," Prosthetics and orthotics international, vol. 28(3), pp. 245-253, Dec. 2004.

[4] H.-N. Ho and L. A. Jones, "Thermal model for hand-object interactions," in Proc. Symposium on Haptic Interface for Virtual Environment and Teleoperator Systems, 2006.

[5] _ _ "Infrared thermal measurement system for evaluating modelbased thermal displays," in Proc. Symposium on Haptic Interface for Virtual Environment and Teleoperator Systems, 2007.

[6] A. Yamamoto, B. Cros, H. Hashimoto, and T. Higuchi, "Control of thermal tactile display based on prediction of contact temperature," in Proc. IEEE international conference on robotics and automation, 2004.

[7] S. Bolanowski, G. Gescheider, R. Verrillo, and C. Checkosky, "Four channels mediate the mechanical aspects of touch," The Journal of the Acoustical Society of America, vol. 84, no. 5, pp. 1680-1694, Nov. 1988.

[8] K. Kim, J. E. Colgate, J. J. Santos-Munné, A. Makhlin, and M. A. Peshkin, "On the design of a tactor for upper extremity prosthetics," Submitted to The IEEE Transactions on Neural Systems and Rehabilitation Engineering.

[9] D. G. Caldwell, N. Tsagarakis, and A. Wardle, "Mechano thermo and proprioceptor feedback for integrated haptic feedback," in Proc. IEEE international conference on robotics and automation, 1997.

[10] I. Darian-Smith, Handbook of Physiology-The Nervous System. American Physiological Soc., 1984.

[11] Omega engineering, inc. [Online]. Available: http://www.omega.com/

[12] D. Kenshalo, T. Decker, and A. Hamilton, "Spatial summation on the forehead, forearm, back produced by radiant and conducted heat," Journal of Comparative and Physiological Psychology, vol. 63, pp. 510-515, 1967. 